\title{
ASSOCIATION BETWEEN ANEMIA, PHYSICAL PERFORMANCE, DEPENDENCY, AND MORTALITY IN OLDER ADULTS IN THE NORTH-WEST REGION OF RUSSIA
}

\author{
A. Turusheva', E. Frolova², E. Korystina'2, D. Zelenukha², P. Tadjibaev², N. Gurina'2, J.M. Degryse ${ }^{1,3}$
}

\begin{abstract}
Objective: To assess the prevalence of anemia and its impact on physical performance, dependency, and mortality in older adults in the north-west region of Russia. Design: A population-based prospective cohort study. Setting: A random sample of the population living in the Kolpino District. Participants: A total of 611 community-dwelling individuals aged 65 years and older. Measurements: Hemoglobin, gender, age, comorbidity, creatinine, C-reactive protein, body mass index, the Mini Nutritional Assessment, the Short Physical Performance Battery, grip strength, the Barthel Index, the Mini-Mental State Examination, and the 15-item Geriatric Depression Scale were measured/administered. A second screening was organized after $33.4 \pm 3$ months. The total observation time was $47 \pm 14.6$ months. Results: The prevalence of anemia was higher in men $(21.4 \%)$ than in women $(18.6 \%)$. After adjustment for age, gender, nutritional status, creatinine levels, mental impairment, and various comorbid conditions, significant associations were found between anemia and dependency [OR $(95 \% \mathrm{CI})=1.798(1.068-3.029) ; \mathrm{p}=0.027]$, lower physical performance [ $\beta(95 \% \mathrm{CI})=-0.717(-1,334--0.100) ; \mathrm{p}=0.023]$, and greater risk of mortality [HR $(95 \% \mathrm{CI})=1.871(1.284--2.728) ; \mathrm{p}=$ 0.001]. Participants with anemia and CRP levels $>5$ had a higher risk of mortality compared with anemic participants with lower CRP levels [HR $(95 \% \mathrm{CI})=3.417(1.869-6.245) ; \mathrm{p}=0.000]$. Conclusion: The estimated prevalence of anemia in the population aged 65 years and older is $19.3 \%(95 \% \mathrm{CI}=15.99-23.13)$. Anemia is associated with poor physical performance and dependency and is an independent predictor of mortality in older individuals.
\end{abstract}

Key words: Anemia, physical performance, dependency, risk mortality, older people.

Abbreviations: BMI: Body mass index; CI: Confidence interval; COPD: Chronic obstructive pulmonary disease; CRP: C-reactive protein; CVD: Cardiovascular disease; GDS15: 15-item Geriartirc Depression Scale; Hb: Hemoglobin; IQR: Interquartile range; HR: Hazard ratio; MCH: Mean corpuscular hemoglobin; MCV; Mean corpuscular volume; MMSE: Mini Mental State Examination; MNA: Mini Nutritional Assessment; OR: odds ratio; SPPB: Short Physical Performance Battery; T0:Date of the first screening; T1:Date of the second screening; T2 The last update of mortality data; WHO: World Health Organisation; USA: United States of America.

\section{Introduction}

The world population is aging rapidly. Since 1980, the number of people aged $60 \mathrm{y}$ and over has doubled to approximately 810 million. The elderly population will continue to grow to approximately 2 billion in 2050. It has been predicted that $22 \%$ of the total population will be older than $60 \mathrm{y}$ and $4.4 \%$ will be older than $80 \mathrm{y}$ in 2050 (1). Unavoidably, this so-called 'gray epidemic' will lead

1. Institut de Recherche Santé et Société Université Catolique de Louvain, Brussels, Belgium; 2. The North-Western State Medical University named after I.I. Mechnikov, St. Petersburg, Russia; 3. Department of Public Health and Primary Health Care, KULeuven, Leuven, Belgium

Corresponding Author: J.M. Degryse, Institut de Recherche Santé et Société Université Catolique de Louvain, Brussels, Belgium, jean-marie.degryse@uclouvain.be to higher burdens of chronic disease, functional decline and disability in many countries challenging health-care and social security programs.

The improved life expectancy in many countries is related to the impressive decrease in mortality from cardiovascular diseases. In contrast to other countries, despite the increase in life expectancy, Russia has higher cardiovascular morbidity (2) and mortality (2) rates and a shorter average life expectancy than those found in Europe and the USA. According to the Federal State Statistics Service of Russia, the average life expectancy in Russia in 2012 was 63.8 years for men and 75.5 years for women (3). According to data from the Ministry of Health of the Russian Federation, the general mortality rate in 2012 was 1,431 per 100,000 people. The mortality 
rate from cardiovascular diseases was 811.7 per 100,000 people, which is twice the rate found in the European Union (EU) (approximately 400 per 100,000) $(4,5)$.

All of these statistics suggest the importance of evaluating people older than 65 years of age in Russia compared with other developed European countries. In a previous report based on the "Crystal" study, it was shown that almost all of the participants had cardiovascular diseases, but none received statins; only some of the participants received antihypertensive drugs and acetylsalicylic acid $(6,7)$. Therefore, the growing population of older individuals who are survivors of cardiovascular mortality without receiving necessary medical and surgical treatment is, from a scientific perspective, a very interesting group for different studies and comparison with other populations. The World Health Organization (WHO) definition of anemia is a hemoglobin concentration $<130 \mathrm{~g} / \mathrm{L}$ in men and $<120$ $\mathrm{g} / \mathrm{L}$ in women. Numerous studies on the prevalence of anemia in the elderly have been published, but the prevalence of anemia is different in each of these studies and is dependent on the type of populations studied, the sample sizes, and the methodologies used. According to WHO data during 1993-2005, the number of individuals suffering from anemia world-wide was 1.62 billion (95\% confidence interval [CI]: $1.50-1.74$ billion), which corresponds to $24.8 \%$ of the population (95\% CI: $22.9 \%$ $26.7 \%)(8)$.

Multiple studies have demonstrated that anemia is an independent risk factor for increased morbidity and mortality, decreased quality of life, depression, dementia, delirium (in hospitalized patients), immune dysfunction, cardiovascular disease, cerebral thrombosis, and decreased bone density (and, consequently, fractures). Anemia has also been associated with a progressive decline in physical performance over time, an increased susceptibility to falling, and frailty in communitydwelling older individuals (9-15).

National data on the prevalence and causes of anemia in the older population in Russia are unavailable (3). Additionally, neither the prevalence, neither die association with adverse outcomes of mild anemia has ever been studied in the older Russian population.

The aim of our study was to assess the prevalence of anemia in general and mild anemia in particular and its impact on physical performance, dependency, and mortality in older adults in the north-west region of Russia, a specific population with a high burden of CVD.

\section{Methods}

The Crystal study is a prospective cohort study of community-dwelling individuals aged 65 years and older living in the Kolpino District of St. Petersburg. A primary care clinic (Policlinic no. 95) serves a population of 58,000 inhabitants based on a territorial concept of administration. Of that population, 10,986 are aged 65 years and older. A random sample of 914 individuals was selected, but 303 people refused to participate before the start of the study. No one was excluded based on health or cognitive function. Selected individuals were invited to participate by telephone. Some people who were unable to come to the Policlinic were examined at home. The local ethics committee of the Medical Academy for Postgraduate Studies approved the study, and informed consent was obtained from all respondents. All data were collected from March to December 2009 (T0). The details regarding sampling and data collection were described in previous publications $(6,7)$.

A second screening (T1) was performed an average of $33.4 \pm 3$ months after the date of the first screening. All patients who survived and consented underwent the same examination that was performed in the first screening.

The average total observation period of the study was $47 \pm 14.6$ months (Figure 1).

\section{Main study parameters}

Hemoglobin was determined using the cyanide-free hemoglobinometry method on the Abbott Cell-Dyn 3700 hematology analyzer. The following normal reference ranges for hemoglobin (venous blood) were used: 130-170 g/L for men and 120-150 g/L for women. All patients with anemia were divided into three groups: mild anemia was defined as a hemoglobin level of 100$119 \mathrm{~g} / \mathrm{L}$ in women and 100-129 g/L in men, moderate anemia as a level of $80-109 \mathrm{~g} / \mathrm{L}$ in both genders, and severe anemia as a level lower than $80 \mathrm{~g} / \mathrm{L}$ in both genders.

\section{Outcome measures}

Mortality data were obtained from the official dataset of Policlinic no. 95. The last update was made in February 2014 (T2).

\section{Physical performance}

The Short Physical Performance Battery (SPPB) consists of timed measures of the following activities: quickly walking, rising from a chair, putting on and taking off a cardigan, and maintaining balance in a tandem stand (15). The activities were timed by specially trained clinical research assistants who were blinded to the laboratory results of the participants. Categories were created for each set of performance measures to allow for analysis of data from participants who were unable to perform a given task. For the walking, chair-stand, and cardigan tests, those who could not complete the tasks were assigned a score of 0 . Those who completed the tasks were assigned a score between 1 and 4 based on the gender- and age-specific quartiles of the distribution 


\section{Figure 1}

Flowchart detailing the data available for survival analysis and the data available for physical and mental decline analysis from the Crystal cohort

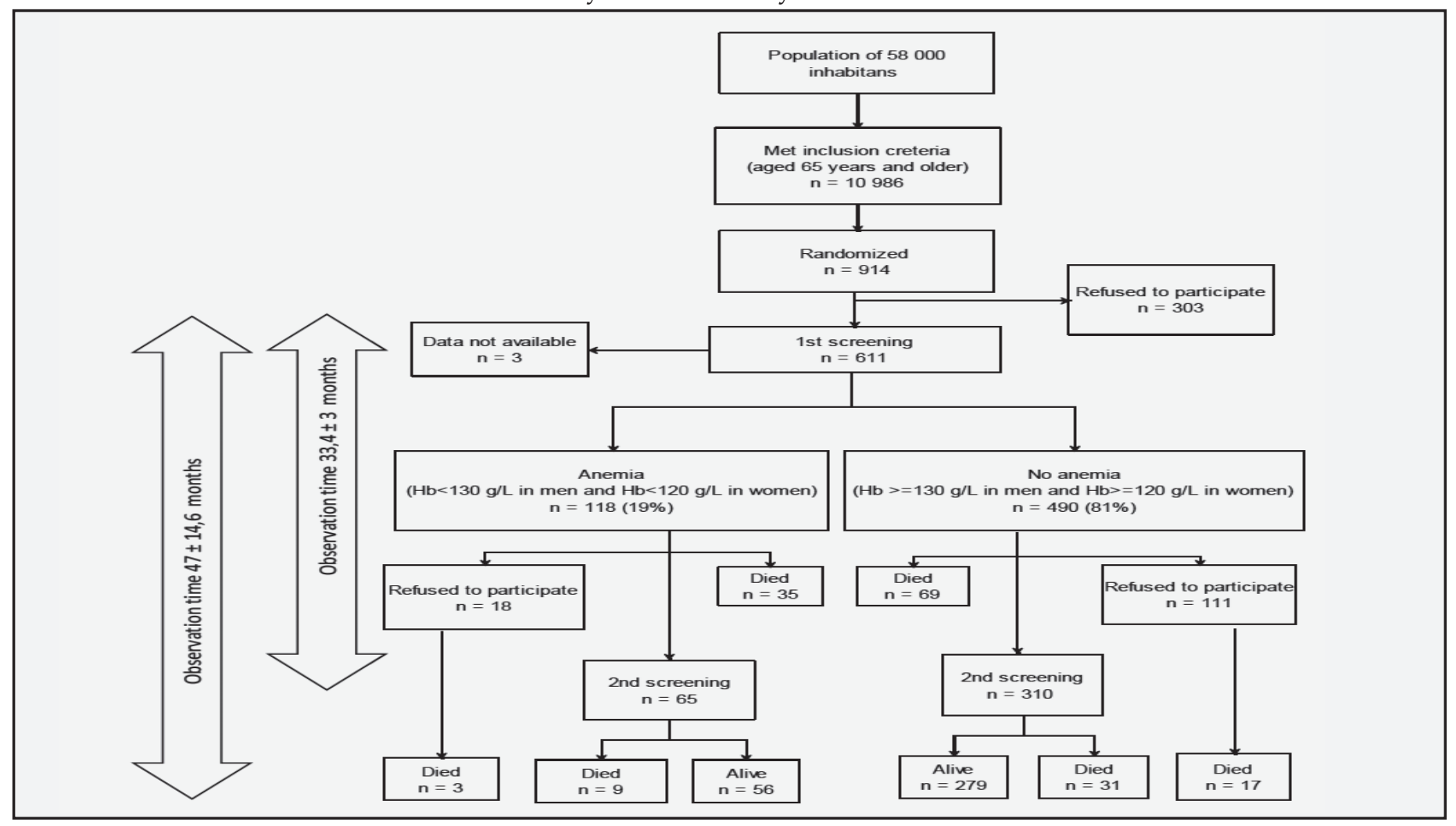

of speed of all participants. A score of 4 corresponded to the highest (fastest) quartile. For the tandem-stand balance test, a score of 0 was assigned to those who were either unable to perform the test or who could only maintain the tandem stand for less than 3 seconds. Those who could maintain a tandem stand for longer than 3 seconds but less than 10 seconds were assigned a score of 1 ; those who could maintain a tandem stand for 10 seconds or more were assigned a score of 2 . A summary performance scale (ranging from $0-14$ ) was created by summing the scores from the individual tests (15-16).

The grip strength test was used to measure the maximum isometric strength of the hand and forearm muscles. The test was conducted using a carpal dynamometer (DK-50, Nizhni Tagil, Russian Federation). The maximum reading $(\mathrm{kg})$ from three attempts for each hand was recorded separately, and the average of the left and right scores was calculated and used for further analysis. The cutoff value used to indicate poor strength was the lowest gender-specific quartile value (17).

\section{Dependency}

The Barthel Index of activities of daily living was used to determine the baseline level of functioning and, consequently, the degree of dependence. The cutoff for dependency was defined as a score of less than 95 (18).

\section{Mental status}

The 15-item Geriatric Depression Scale (GDS-15) was used to screen for depressive symptoms. A person with a total score greater than 5 is considered to be at risk for depression (19-20).

The Mini-Mental State Examination (MMSE) was used to determine cognitive impairment. The cutoff value for cognitive ability is 24, but it is also useful to study the degree of impairment. Therefore, participants were classified into four categories (25-30, normal mental status; 21-24, mild cognitive impairment; 10-20, moderate cognitive impairment; and $0-9$, severe cognitive impairment) (21).

\section{Mental and physical decline}

Relevant declines in the MMSE, SPBB, and average grip strength scores from both hands were determined using the Edwards-Nunnally index (22). This index is used to determine the probability of a substantial individual change and avoids the problem of regression to the mean. Based on the scale reliability and the $95 \%$ $\mathrm{CI}$ of the mean score at T0, the index is used to assess whether a significant change has occurred between T0 and T1. Subjects who shifted from GDS- $15<5$ at baseline to GDS-15 $\geq 5$ at T1 were defined as having a significant worsening in depression status. New incident 
occurrences of dependency were defined as shifts in Barthel index scores to less than 95.

Physical decline was considered to be present when a study participant showed a significant decline in the score of at least one of the three tests (the Barthel Index of activities of daily living, the SPBB, and average grip strength of both hands). Mental decline was considered to be present when a participant presented a decline in the score of at least one of the two tests (the MMSE or GDS test).

\section{Covariates}

\section{Nutritional status}

Nutritional status was evaluated using the Mini Nutritional Assessment (MNA) questionnaire and body mass index (BMI) $(23,24)$. Weight was measured using either a stationary calibrated medical scale at the Policlinic or a calibrated bathroom scale at home. The cutoff value used in the analysis of BMI was $23 \mathrm{~kg} / \mathrm{m}^{2}$. A MNA score between 17.0 and 23.5 was interpreted as indicating a risk for malnutrition, and a score less than 17.0 was considered an indicator of frank malnutrition.

\section{Comorbidity}

Details of past and current medical problems were collected based on anamnesis or information presented in the medical records. Information on angina pectoris, myocardial infarction, arrhythmias, peripheral artery disease, stroke, obstructive pulmonary disease or asthma, diabetes mellitus, cancer, osteoarthritis, and rheumatoid arthritis was systematically documented. A disease count was used as an index of comorbidity.

\section{Laboratory tests}

Laboratory tests included measures of creatinine and C-reactive protein (CRP) levels. Creatinine was determined by the Jaffe reaction method using the Roche Hitachi 912 chemistry analyzer. CRP was determined by the immunoturbidimetric method using the Roche Hitachi 912 chemistry analyzer. The following normal reference ranges for each laboratory test were used: hemoglobin (venous blood), $130-170 \mathrm{~g} / \mathrm{L}$ for men and 120 - $150 \mathrm{~g} / \mathrm{L}$ for women; creatinine, $53-106 \mathrm{mmol} / \mathrm{L}$ for men and $44-88 \mathrm{mmol} / \mathrm{L}$ for women; CRP, $0-5 \mathrm{mg} / \mathrm{L}$ for both sexes.

\section{Statistical analyses}

All statistical calculations were performed using the SPSS 20.0 (SPSS Inc., Chicago, IL, USA) and MedCalc 11.5.00 (Medcalc Software, Oostende) software. Desciptive statistics are presented as the mean \pm standard deviation (SD) or median with inter-quartile range [IQR].
Differences between participants with and without anemia at baseline were compared using Student's $t$ test and the Mann-Whitney U test (for continuous variables) or chi-square test (for categorical variables). To assess the correlation of anemia with physical performance at baseline taking into account the influence of gender, nutritional status, and comorbidity, we used linear regression models. In the first model, we estimated the association of age and gender with the SPPB scores used as a dependent variable. The second model included the same variables as the first model, but we added creatinine and the assessment of nutritional status. In the third model, we added adjustments for the MMSE score and co-morbidities. The fourth model included additional adjustment for high CRP levels. To assess the association of anemia with the Barthel Index (<95), we used multiple logistic regression with models similar to those used for the Physical Performance Battery. Variables were first checked for multicollinearity.

Kaplan-Meier curves were used to visualize the survival analysis, and significance was evaluated using the log-rank test. We used Cox proportional hazard models to investigate the association between prevalent anemia at baseline and mortality with adjustments for the same models as those used to estimate physical function and dependency in basic activities of daily living.

\section{Results}

\section{The prevalence of anemia}

Blood samples from 608 subjects were available for analysis at baseline (fig 1). Thirty-seven men and 81 women met the criteria for anemia, which corresponded to $19.3 \%(95 \% \mathrm{CI}=15.99-23.13)$ of the population aged 65 years and older. A total of $113(96 \%)$ participants had mild anemia, $4(3 \%)$ had moderate anemia, and $1(1 \%)$ had severe anemia. The prevalences of anemia among men and women were $21.4 \%$ and $18.6 \%$, respectively, but this different was not significant. The mean $( \pm \mathrm{SD})$ serum hemoglobin level was $120( \pm 6.79) \mathrm{g} / \mathrm{L}$ in men with anemia and $145( \pm 10.66) \mathrm{g} / \mathrm{L}$ in men without anemia. The mean $( \pm \mathrm{SD})$ serum hemoglobin level in women with anemia was lower $(108( \pm 10.84) \mathrm{g} / \mathrm{L})$ than that in men $(134( \pm 9.86) \mathrm{g} / \mathrm{L})$, but this difference was not significant $(p=0.038)$.

We noted an increased prevalence of anemia among both men and women with increasing age. The prevalence of anemia in the various age groups were as follows: $65-69$ years, $14 \%[95 \% \mathrm{Cl}=8-22]$; $70-74$ years, $17.9 \%[95 \% \mathrm{Cl}=12-25] ; 75-79$ years, $20.6 \%[95 \% \mathrm{Cl}=$ 14-29]; 80-84 years, $22.1 \%$ [95\% $\mathrm{Cl}=14-33$ ]; 85-89 years, $31.7 \%[95 \% \mathrm{Cl}=16-54]$; and 90 years and older, $16.7 \%$ [ $95 \% \mathrm{Cl}=0.4-0.93]$. 
Table 1

Health characteristics of subjects with and without anemia

\begin{tabular}{|c|c|c|c|}
\hline Characteristic & $\begin{array}{l}\text { Anemia } \\
\mathrm{n}=118(19.4 \%)\end{array}$ & $\begin{array}{l}\text { No anemia } \\
\mathrm{n}=490 \\
(80.6 \%)\end{array}$ & $\mathrm{p}$ \\
\hline Age, median (IQR) & $77(71-81)$ & $74(70-79)$ & \\
\hline \multicolumn{4}{|l|}{ Gender, $\mathrm{n}(\%)$} \\
\hline Female & $81(18.6)$ & $354(81.4)$ & \\
\hline Male & $37(21.4)$ & $136(78.6)$ & \\
\hline \multicolumn{4}{|l|}{ MNA, n, \% } \\
\hline$<17$ «Malnourished" & $6(5.1)$ & $4(0.8)$ & $0.001^{*}$ \\
\hline 17-23.5 «At risk of malnutrition» & $26(22.0)$ & $79(16.1)$ & $0.011^{*}$ \\
\hline$>23.5$ «Normal nutritional status» & $86(72.9)$ & $407(83.1)$ & \\
\hline $\mathrm{BMI}$, mean $\pm \mathrm{SD}$ & $26.9 \pm 5.0$ & $28.9 \pm 4.8$ & \\
\hline Creatinine, median (IQR) & $78(64-94)$ & $76(66-86)$ & \\
\hline $\begin{array}{l}\text { Number of comorbidities, median } \\
\text { (IQR) }\end{array}$ & $3(2-4)$ & $3(2-3)$ & \\
\hline Coronary artery disease, $\mathrm{n}(\%)$ & $97(78.9)$ & $382(78.8)$ & \\
\hline Myocardial infarction, $\mathrm{n}(\%)$ & $15(12.2)$ & $62(12.8)$ & \\
\hline Diabetes mellitus, n (\%) & $14(11.4)$ & $72(14.8)$ & \\
\hline COPD, n (\%) & $31(25.2)$ & $109(22.5)$ & \\
\hline Asthma, n (\%) & $9(7.6)$ & $18(3.7)$ & \\
\hline Peripheral arterial disease, $\mathrm{n}(\%)$ & $28(22.8)$ & $106(21.6)$ & \\
\hline Atrial fibrillation, $\mathrm{n}(\%)$ & $44(35.8)$ & $141(29.1)$ & \\
\hline Arthritis or arthritis (\%) & $8(6.5)$ & $15(3.1)$ & \\
\hline Stroke, n (\%) & $15(12.7)$ & $75(15.3)$ & \\
\hline High blood pressure, $\mathrm{n}(\%)$ & $116(94.0)$ & $448(92.4)$ & \\
\hline \multicolumn{4}{|l|}{ MMSE, n (\%) } \\
\hline 25-30 Normal & $75(63.6)$ & $323(65.9)$ & \\
\hline 21-24 Mild & $20(16.9)$ & $104(21.2)$ & \\
\hline 10-20 Moderate & $21(17.8)$ & 57 (11.6) & \\
\hline 0-9 Severe & $2(1.7)$ & $6(1.2)$ & \\
\hline $\mathrm{CRP}>5 \mathrm{mg} / \mathrm{L}, \mathrm{n}(\%)$ & $24(20.3)$ & 57 (11.6) & $0.035^{*}$ \\
\hline
\end{tabular}

*- comparisons were made using the chi-square test; MNA - Mini nutritional assessment; BMI - Body mass index; Multimorbidity - several comorbidities in one person; COPD - Chronic obstructive pulmonary disease; MMSE - Mini-mental state examination; CRP - C-reactive protein

\section{The impact of anemia on outcomes}

Individuals with anemia showed significantly worse SPBB scores than those without anemia. Those with lower physical performance scores were more likely to have signs of malnutrition or be at risk for malnutrition, to have cognitive impairment, and to be older. The association between lower physical performance and anemia remained significant after adjustments were made for all of these covariates $[\beta(95 \% \mathrm{CI})=-0.717$ $(-1.334-0.100) ; \mathrm{p}=0.023]$. However, this association disappeared after adjustment for CRP levels $[\beta(95 \% \mathrm{CI})=$ -0.507 (-1.155-0.140); $\mathrm{p}=0.125]$ (Table 2).

Participants with anemia had a greater risk of dependency in basic activities of daily living compared with participants without anemia at baseline. After we adjusted for gender, comorbidities, nutrition, and mental status, as well as creatinine and CRP levels, we found that anemia was still significantly associated with a higher risk of dependency [OR $(95 \% \mathrm{CI})=1.798(1.068-3.029)$; $\mathrm{p}$ $=0.027$ ] (Table 3)

Anemia at baseline did not appear to predict a decline in physical [OR $(95 \% \mathrm{CI})=1.810(0.880-3.723) ; \mathrm{p}=0.107]$ and mental [OR $(95 \% \mathrm{CI})=0.877(0.486-1.581) ; \mathrm{p}=0.662$ ] functions over an observation time of $34 \pm 3$ months.

The mortality rates at $\mathrm{T} 2$ of the participants with and without anemia were $39.8 \%(n=47)$ and $23.9 \%$ $(\mathrm{n}=117)$, respectively. Kaplan-Meier curves showed a higher cumulative survival for all-cause mortality for subjects without anemia (log-rank $=<0.001$ ) (fig 2). Anemia remained significantly associated with all-cause mortality, with a 1.87-fold increase in the risk for allcause mortality after adjustment for various confounding factors, including age, gender, nutritional status, renal function, comorbidity, cognitive impairment, and CRP levels [HR $(95 \% \mathrm{CI})=1.871(1.284-2.728) ; \mathrm{p}=0.001$ ] (Table 4).

Moreover, we observed that participants with anemia and high CRP levels had a significantly higher mortality risk compared with anemic participants with low CRP levels at baseline (fig 3). This impact of anemia combined with high CRP levels remained significant after adjustment for potential confounders in the respective models [HR $(95 \% \mathrm{CI})=3.143(1.773-5.571) ; \mathrm{p}=0.000]$ (Table 5).

\section{Covariates}

Individuals with anemia were more likely to have signs of malnutrition or to be at risk for malnutrition and higher CRP levels than those without anemia. The prevalence of anemia was $18.9 \%$ in the group of participants with CRP $>5 \mathrm{mg} / \mathrm{L}$ and $29.6 \%$ in the group of participants with CRP $\leq 5 \mathrm{mg} / \mathrm{L}(\mathrm{p}=0.035)$. We found no significant difference in the mean $( \pm S D)$ hemoglobin level among participants with anemia and high CRP levels $(109( \pm 14.92) \mathrm{g} / \mathrm{L})$ and those with anemia and normal CRP levels $(113( \pm 9.98) \mathrm{g} / \mathrm{L})(\mathrm{p}=0.125)$.

No significant differences in BMI and the prevalence of chronic diseases, CVD, and cognitive impairment in the groups of participants with and without anemia were observed (Table 1). The prevalence of CVD at baseline among participants with anemia and high CRP levels $(87.5 \%[95 \% \mathrm{Cl}=54-133 \%])$ compared with that among participants with anemia and normal CRP levels (85\% $[95 \% \mathrm{Cl}=66-108 \%])$ was also the same $(\mathrm{p}=0.971)$.

The incidence rate of new cases of cardiovascular events (myocardial infarction, stroke, and atrial fibrillation) over the $33.3 \pm 4$-month observation period was 0.176 [95\% CI $=0.132-0.229]$ in the group of people without anemia at baseline was not significantly different from the incidenc in the group of people with anemia at baseline 0.092 [95\% CI $=0.034-0.201]$. 
Table 2

Association between physical performance and anemia

\begin{tabular}{|c|c|c|c|c|c|c|c|c|}
\hline & Model 1 & & Model 2 & & Model 3 & & Model 4 & \\
\hline & $\beta(95 \% \mathrm{CI})$ & $\mathrm{p}$ & $\beta(95 \% \mathrm{CI})$ & $\mathrm{p}$ & $\beta(95 \% \mathrm{CI})$ & $\mathrm{p}$ & $\beta(95 \% \mathrm{CI})$ & $\mathrm{p}$ \\
\hline Anemia & $-0.786(-1.426--0.147)$ & .016 & $-0.716(-1.339--.093)$ & .024 & $-.717(-1.334--0.100)$ & .023 & $-.507(-1.155-0.140)$ & .125 \\
\hline Gender & $0.151(-0.413-0.715)$ & .598 & $0.025(-0.553-0.603)$ & .932 & $.038(-0.534-0.611)$ & .896 & $.186(-0.440-0.812)$ & .560 \\
\hline Age & $-.211(-0.254--0.168)$ & .000 & $-0.204(-0.247--0.162)$ & .000 & $-.178(-0.222--0.133)$ & .000 & $-.179(-0.226--0.132)$ & .000 \\
\hline MNA & & & $-1.855(-2.487--1.224)$ & .000 & $-1.600(-2.238--0.961)$ & .000 & $-1.946(-2.629--1.263)$ & .000 \\
\hline Creatinine & & & $0.003(-0.009-0.015)$ & .591 & $.003(-0.008-0.015)$ & .578 & $.002(-0.012-0.016)$ & .793 \\
\hline Multimorbidity & & & & & $-.056(-0.248-0.137)$ & .571 & $-.134(-0.342-0.074)$ & .206 \\
\hline MMSE & & & & & $-.639(-0.987--0.291)$ & .000 & $-.631(-1.005--0.258)$ & .001 \\
\hline $\mathrm{CRP}>5(\mathrm{mg} / \mathrm{L})$ & & & & & & & $-.917(-1.655--.179)$ & .125 \\
\hline
\end{tabular}

MNA - Mini nutritional assessment; Multimorbidity - several comorbidities in one person; MMSE - Mini-mental state examination; CRP - C-reactive protein; Model 1 . Unadjusted + age, gender; Model 2: Model 1 + MNA + creatinine level; Model 3: Model 2 + MMSE; Model 4: Model 3 + CRP level

\section{Table 3}

Association between dependency (defined as Barthel Index >95) and anemia

\begin{tabular}{|c|c|c|c|c|c|c|c|c|}
\hline & Model 1 & & Model 2 & & Model 3 & & Model 4 & \\
\hline & OR $(95 \% \mathrm{CI})$ & $\mathrm{p}$ & OR $(95 \% \mathrm{CI})$ & $\mathrm{p}$ & OR $(95 \% \mathrm{CI})$ & $\mathrm{p}$ & OR $(95 \% \mathrm{CI})$ & $\mathrm{p}$ \\
\hline Anemia & $1.852(1.175-2.919)$ & .008 & $1.717(1.066-2.767)$ & .026 & $1.761(1.075-2.885)$ & .025 & $1.798(1.068-3.029)$ & .027 \\
\hline Gender & $1.092(.695-1.716)$ & .703 & $1.203(.733-1.974)$ & .466 & $1.226(.737-2.040)$ & .433 & $1.227(.704-2.139)$ & .471 \\
\hline Age & $1.101(1.065-1.138)$ & .000 & $1.096(1.058-1.135)$ & .000 & $1.070(1.030-1.111)$ & .000 & $1.063(1.022-1.107)$ & .003 \\
\hline MNA & & & $.311(.197-.492)$ & .000 & $.386(.239-.624)$ & .000 & $.347(.207-.582)$ & .000 \\
\hline Creatinine & & & $1.001(.992-1.010)$ & .875 & $1.001(.991-1.011)$ & .855 & $1.002(.991-1.014)$ & .671 \\
\hline Multimorbidity & & & & & $1.131(.961-1.332)$ & .139 & $1.040(.871-1.243)$ & .663 \\
\hline MMSE & & & & & $1.834(1.405-2.394)$ & .000 & $1.715(1.279-2.300)$ & .000 \\
\hline $\mathrm{CRP}>5(\mathrm{mg} / \mathrm{L})$ & & & & & & & $.628(.352-1.123)$ & .117 \\
\hline
\end{tabular}

MNA - Mini nutritional assessment; Multimorbidity - several comorbidities in one person; MMSE - Mini-mental state examination; CRP - C-reactive protein. Model 1: Unadjusted + age, gender; Model 2: Model 1 + MNA + creatinine level; Model 3: Model 2 + MMSE; Model 4: Model 3 + CRP level

\section{Figure 2}

Kaplan-Meier curves for survival based on anemia of older adults in the north-west region of Russia

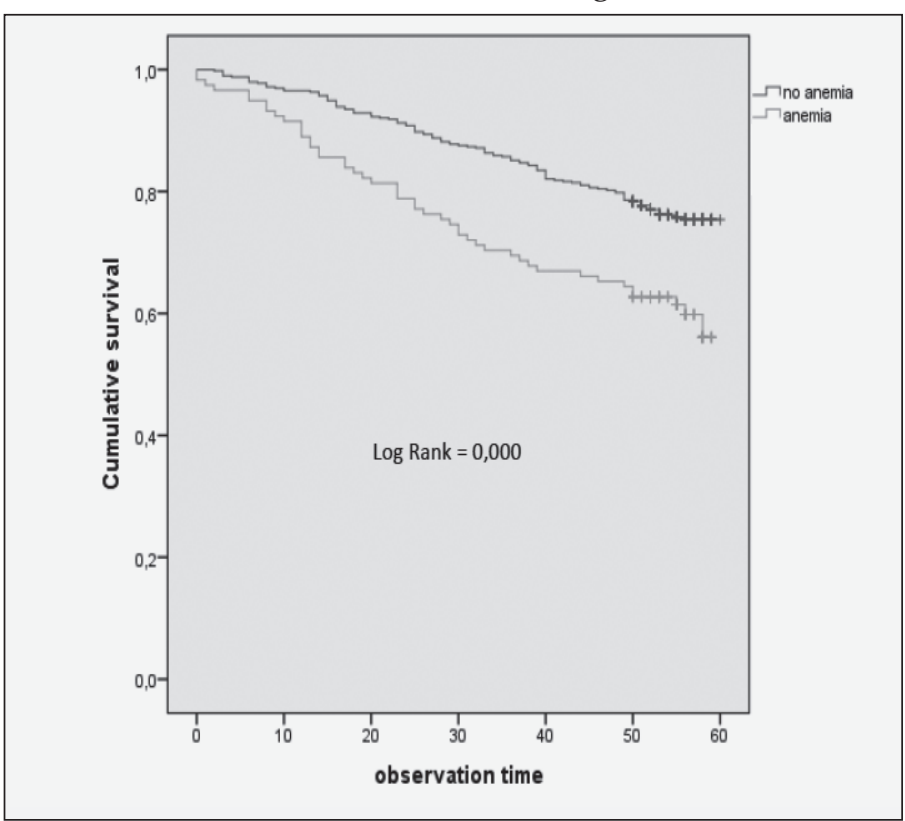

\section{Main findings}

\section{The prevalence of anemia}

The Crystal study is the first prospective cohort study of community-dwelling individuals in the Russian Federation that has investigated the prevalence of anemia. Using the WHO criteria, an anemia prevalence of $19 \%$ was identified in this cohort.

\section{Discussion}

\section{The impact of anemia on outcomes}

We found a significant association of anemia with dependency and lower physical performance at baseline, as well as a greater risk of mortality and anemia of follow-up. These associations remained significant after adjustment for age, gender, nutritional status, creatinine levels, mental impairment, high CRP and various comorbid conditions. 
Table 4

Anemia as a predictor of mortality (Cox proportional hazard analysis)

\begin{tabular}{|c|c|c|c|c|c|c|c|c|}
\hline & Model 1 & & Model 2 & & Model 3 & & Model 4 & \\
\hline & HR $(95 \%$ CI) & $\mathrm{p}$ & HR $(95 \%$ CI) & $\mathrm{p}$ & HR $(95 \%$ CI) & $\mathbf{p}$ & HE $(95 \% \mathrm{CI})$ & $\mathrm{p}$ \\
\hline Anemia & $1.614(1.147-2.270)$ & .006 & $1.526(1.076-2.162)$ & .018 & $1.761(1.075-2.885)$ & .004 & $1.871(1.284-2.728)$ & .001 \\
\hline Gender & $.539(.386-.751)$ & .000 & $.567(.398-.807)$ & .002 & $.570(.399-.813)$ & .002 & $.579(.387-.868)$ & .008 \\
\hline Age & $1.115(1.087-1.144)$ & .000 & $1.114(1.084-1.144)$ & .000 & $1.094(1.064-1.125)$ & .000 & $1.087(1.055-1.119)$ & .000 \\
\hline MNA & & & $.606(.426-.862)$ & .005 & $.680(.476-.971)$ & .034 & $.716(.483-1.061)$ & .096 \\
\hline Creatinine & & & $1.004(.998-1.010)$ & .018 & $1.004(.998-1.010)$ & .226 & $1.005(.997-1.013)$ & .253 \\
\hline Multimorbidity & & & & & $.947(.837-1.071)$ & .384 & $.915(.796-1.053)$ & .215 \\
\hline MMSE & & & & & $1.500(1.244-1.808)$ & .000 & $1.575(1.280-1.939)$ & .000 \\
\hline $\mathrm{CRP}>5(\mathrm{mg} / \mathrm{L})$ & & & & & & & $.672(.443-1.017)$ & .060 \\
\hline
\end{tabular}

MNA - Mini nutritional assessment; Multimorbidity - a number of comorbidities in one person; MMSE - Mini-mental state examination; CRP - C-reactive protein; Mode 1: Unadjusted + age, gender; Model 2: Model 1 + MNA + creatinine level; Model 3: Model 2 + MMSE; Model 4: Model 3 + CRP level

Table 5

Anemia + high CRP levels as predictors of mortality (Cox proportional hazard analysis)

\begin{tabular}{|c|c|c|c|c|c|c|}
\hline & Model 1 & & Model 2 & & Model 3 & \\
\hline & HR $(95 \%$ CI) & $\mathrm{p}$ & HR $(95 \%$ CI) & $\mathrm{p}$ & HR $(95 \%$ CI) & $\mathrm{p}$ \\
\hline Anemia $+\mathrm{CRP}>5 \mathrm{~g} / \mathrm{L}$ & $3.927(2.231-6.912)$ & .003 & $3.341(1.849-6.037)$ & .000 & $3.417(1.869-6.245)$ & .000 \\
\hline Gender & $.506(.347-.738)$ & .000 & $.554(.370-.828)$ & .004 & $.570(.380-.856)$ & .007 \\
\hline Age & $1.111(1.080-1.142)$ & .000 & $1.109(1.078-1.141)$ & .000 & $1.087(1.055-1.119)$ & .000 \\
\hline MNA & & & $.656(.443-.970)$ & .035 & $.731(.491-1.087)$ & .122 \\
\hline Creatinine & & & $1.005(.997-1.013)$ & .215 & $1.004(.996-1.012)$ & .313 \\
\hline Multimorbidity & & & & & $.913(.794-1.050)$ & .204 \\
\hline MMSE & & & & & $1.588(1.288-1.957)$ & .000 \\
\hline
\end{tabular}

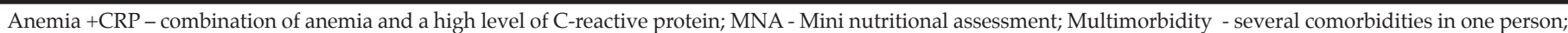
MMSE - Mini-mental state examination; Model 1: Unadjusted + age, gender; Model 2: Model 1 + MNA + creatinine level; Model 3: Model 2 +

\section{Figure 3}

Cumulative survival according to the presence of anemia and high CRP levels

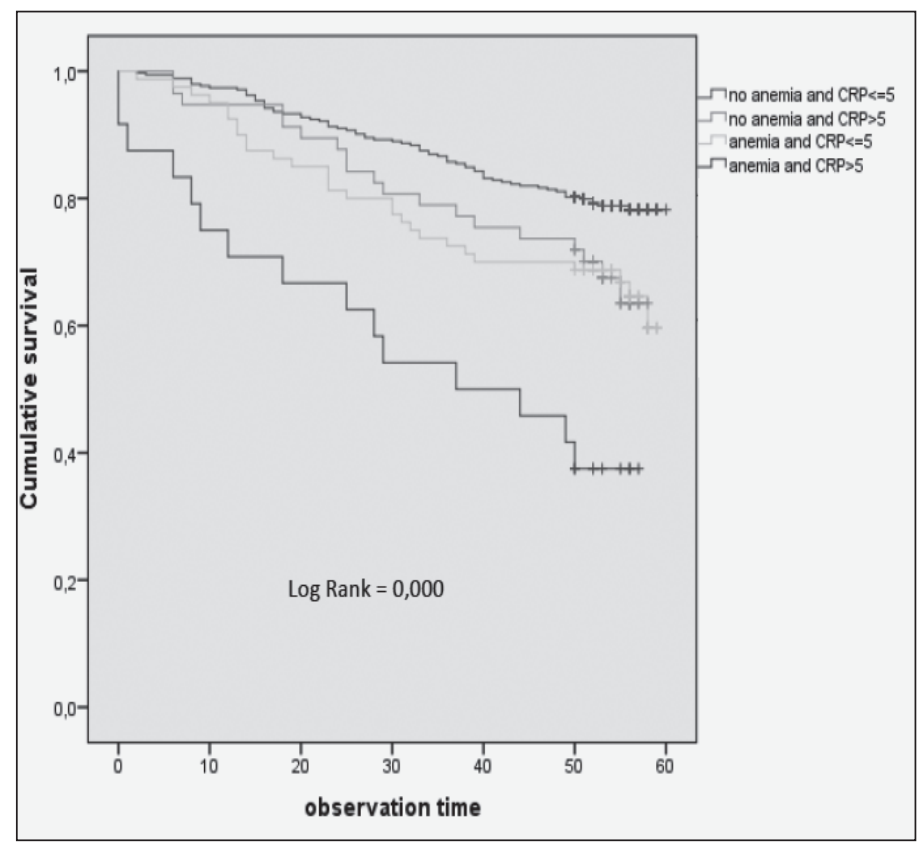

\section{Interpretation of findings in relation to previously published studies}

The prevalence of anemia

Anemia is a common concern in geriatric health, but estimates of the prevalence of this condition vary substantially. In a systematic review by C. Beghe and colleagues, the findings of large-scale studies revealed the prevalence of anemia in older men $(2.9 \%-61 \%)$ to be higher than that in older women $(3.3 \%-41 \%)(9)$. This variability is related to several factors, including the setting of the study, the health status of the subject population, and the criteria used to define anemia. The InCHIANTI (25) and KORA-Age (26) studies were performed in a sample of community-dwelling people aged 65 years and older and had inclusion criteria similar to those of the Crystal study. These studies indicated a slightly lower prevalence of anemia than that found in the current study. The prevalence of anemia was $12 \%$ in the InCHIANTI study (25) and $17.7 \%$ in the KORA-Age study (25). In this study, as in the KORA-Age study (26), the prevalence of anemia was slightly higher in men 40 
(21.4\%) than in woman (18.6\%), although this difference was not significant.

We observed an increased prevalence of anemia with age. The large-scale studies showed the same results, usually with a rise in prevalence after the age of 85 years (9).

\section{Mild anemia as a risk factor}

In the present study, the vast majority of anemia cases were mild. These findings are consistent with the results of other researchers (11,25-31). Mild anemia is a frequent laboratory finding in the elderly but is usually disregarded in everyday practice as inconsequential (28, 30). Until recently, anemia was often considered a normal consequence of aging with no influence on overall health (32).

In older people, aging alone is unlikely to cause anemia. Many underlying conditions can lead to anemia, but the most common conditions are chronic inflammation, chronic renal failure, nutrient deficiencies, and age-specific changes in the hematopoietic system $(9,29,32)$. We found that individuals with anemia were more likely to have signs of malnutrition or to be at risk for malnutrition and to have higher creatinine and CRP levels than those without anemia. All of these states can lead to an increased risk of mortality and other adverse outcomes; for this reason, we considered these factors as confounders. In the current study, mild anemia was associated with dependency, lower physical performance, and a greater risk of mortality in people 65 years and older. These findings confirm the results of previous studies involving people 65 years and older living in communities in other countries $(9,16,27,30-35)$.

\section{How is anemia associated with worse physical performance and dependency?}

There are several existing hypotheses to explain this relationship. Considering that fatigue is often a chief complaint among anemic patients, it is conceivable that older adults with anemia decrease their physical activity and lose muscle strength and mass through disuse (36). Furthermore, muscle strength, muscle mass, and density, measured by computed tomography, were significantly lower in anemic compared with non-anemic communitydwelling older adults $(36,37)$. In addition, a decreased hemoglobin concentration can reduce the oxygenation of muscles, particularly in older adults with a greater vascular disease burden, which can impair tissue perfusion). Another possibility is that the association of anemia with physical function outcomes in older adults may reflect chronic inflammation or decreased testosterone levels, both of which are factors that can adversely affect erythropoiesis and muscle mass (36-38).

Cytokines are markers of inflammation and are involved in numerous physiological functions, such as immunoregulation, hematopoiesis, tissue homeostasis, and catabolic processes and in the production of CRP. Therefore, it is difficult to indicate a single possible mechanism to explain their potential effects on physical performance (38-40). Nonetheless, several previous studies identified a significant association between high levels of CRP and Il6 and a loss of skeletal muscle mass and decreased muscle strength in older people $(36,39$, 40). However, Brinkley and colleagues indicated that inflammatory biomarkers have an effect on physical function that is independent of age, gender, race, and body composition (41).

A unique finding of this study was the decreased association between anemia and the SPBB score after adjustment for high CRP levels. A possible explanation may be a stronger association of decreased muscle strength with high CRP levels than with anemia in older people.

We did not observe a significantly different degree of decline in physical and mental functions over the $33.4 \pm 3$-month observation period between anemic and non-anemic subjects. This result may be attributed to the relatively short observation period. For example, the duration of the EPESE study (11) was 4 years. In this study of individuals aged 65 years and older, mild anemia was associated with a significantly greater decline in physical performance over 4 years. These associations were not explained by baseline diseases or by low serum cholesterol, iron, or albumin levels. Anemia was also associated with subsequent physical decline individuals without co-morbidity (cancer, infectious diseases, and renal failure) at baseline (11).

We found that participants with anemia had a significantly higher mortality risk compared with nonanemic participants at baseline.

This finding confirmed the results of previous studies involving older people living in the community and remained significant even after adjustment for comorbid conditions (e.g., cardiovascular disease, cancer, kidney disease) $(9,27,28,35,37)$. Noteworthy, even low to normal hemoglobin concentrations were associated with increased mortality, although this finding is not consistent across studies and depends on the definition of low to normal concentrations, the comparison group, race/ethnicity, and comorbidities $(16,35)$. Thus, Culleton and colleagues suggested that the optimal hemoglobin level to avoid hospitalization and mortality should be 130-150 g/L for women and 140-170 g/L for men (42).

The specific mechanisms by which anemia may adversely affect relevant health-related outcomes in the elderly are unknown (37). In theory, anemia interferes with the delivery of oxygen to the brain, heart, and muscles (37) and leads to hemodynamic stresses related to increased cardiac output, which, if sustained, leads to left ventricular enlargement and an increased risk of cardiovascular events (27). A background of 
atherosclerosis exacerbates these processes. However, an alternative explanation is that anemia may be a consequence of the underlying comorbid diseases and frailty that cause disability (37). Addressing this question is critical for geriatric research. Because the prevention and management of disability is a major goal of geriatric medicine, the possibility that anemia is one of the few potentially reversible causes of disability in older individuals is particularly appealing.

We found that participants with anemia and high CRP levels had a significantly higher mortality risk compared with anemic participants with low CRP levels at baseline.

Some studies have suggested that inflammatory markers are independent predictors of cardiovascular events in older individuals $(40,42,43)$. In a recent metaanalysis (43), high CRP levels were found to be associated with the risk of coronary heart disease, ischemic stroke, and death from vascular and non-vascular diseases (including several cancers and respiratory diseases), even in individuals without initial vascular disease. This association with ischemic vascular disease depends considerably on conventional risk factors and other markers of inflammation (43).

In Russia, the mortality rate from cardiovascular diseases is two times higher than that in the European Union, and a majority of the participants in our study did not receive the necessary medical and surgical treatments for CVD. Therefore, it would be interesting to study the association between the prevalence of CVD, anemia, and mortality in this population. In the current study, anemia combined with high CRP levels was significantly associated with mortality from all causes after adjustment for age, gender, nutritional status, creatinine levels, mental decline, and various comorbid conditions, including CVD. Additionally, we found no significant difference between the prevalence of CVD at baseline and new cardiovascular events (e.g., myocardial infarction, stroke, and atrial fibrillation) during all observation times in individuals with high and normal CRP levels.

Thus, we can assume that the presence of even mild anemia in combination with a high level of CRP in individuals aged 65 years and older is independently associated with a threefold increased risk of death by all causes without any link to CVD. This suggestion agrees with findings of the Cardiovascular Health Study (44) and the Iowa 65+ Rural Health Study (45). A stronger association between the combination of anemia and high CRP levels and mortality may be linked to the cumulative negative effects of anemia and high CRP levels or to undiagnosed underlying subclinical diseases that cause the development of anemia and increased inflammationsuppressing erythropoiesis.

\section{Strengths and limitations}

Our study had several strengths. All participants were selected using the random sampling method. To our knowledge, the Crystal study is the first study to assess the prevalence of anemia and its impact on physical performance and mortality in community-dwelling individuals aged 65 years and older in this part of the Russian Federation.

The short period of time between the first and second screening (33.4 \pm 3 months) may be considered a potential limitation of our study. This may be one of the reasons for the lack of association between anemia and physical and mental decline.

Another limitation of our study could be that MCV, $\mathrm{MCH}$, vitamin B12, folate, and iron levels were not measured at baseline. Therefore, we cannot judge what the causes of anemia were in this population. Another limitation is the lack of information about hospitalization and the exact causes of death.

\section{Implications of our results}

Anemia should be taken into account in geriatric assessments and should be evaluated as a risk factor of mortality, dependency, and poor physical performance. Further research on the causes of anemia in this age group is needed. Additional interventional studies are needed to produce evidence that treatment of mild anemia improves health outcomes before screening of anemia will become part of national clinical guidelines.

\section{Conclusions}

The prevalence of anemia in the population living in the North-West region of Russia aged 65 years and older is $19.3 \%$ (95\% CI $=15.99-23.13)$. Mild anemia is an independent predictor of poor physical performance, dependency, and increased mortality risk in older individuals.

Acknowledgment: The authors would like to express their appreciation to the head of the polyclinic no. 95 Tatyana Isaeva, and to the chief nurses Irina Yakovleva and Irina Dobritsa for their input to the organization and management of the study.

Ethical approval: The protocol of the study was approved by the local medical ethics review board (MAPS protocol N 17 from 05.11.2008).

Funding: The President of the Russian Federation (Grant 192-RP) supported this work.

Conflict of Interest: none to declare

\section{References}

1. United Nations, Department of Economic and Social Affairs, Population Division. World Population Ageing 2013; ST / ESA/SER.A/348.

2. World health organization regional office for Europe (2012) Executive summary of the European health report 2012: Moving Europe towards health and well-being. Malta http:/ / www.euro.who.int/_data/assets/ pdf_file/0017/170108/RC62-id01-Eng.pdf Accessed 15 May 2014

3. The Federal State Statistics Service of Russia. Official data about the population of the Russian Federation distributed 2013. http://www.gks. $\mathrm{ru} / \mathrm{wps} / \mathrm{wcm} /$ connect/rosstat_main/rosstat/ru/statistics/population/ Accessed 15 May 2014

4. Yakovleva TV. Report of vice-minister of Health of the Russian Federation: 
Improving delivery of medical care cardiovascular diseases in the Russian Federation 2012. http: / / old.rosminzdrav.ru/health/ cardiovascular Accessed 15 May 2014

5. European Heart Network and European Society of Cardiology. European Cardiovascular Disease Statistics 2012 edition http: / / www.escardio.org/ about/documents/eu-cardiovascular-disease-statistics-2012.pdf Accessed 15 May 2014

6. Gurina NA, Frolova EV, Degryse JM. Examine the health status of older people in one of the districts of the St. Petersburg: the results of The "Crystal" Study. J Advances in gerontology 2012; 24 (1): 114-120.

7. Gurina NA, Frolova EV, Degryse JM. A Roadmap of Aging in Russia: The Prevalence of Frailty in Community-Dwelling Older Adults in the St. Petersburg District - The "Crystal" Study. Am Geriatr Soc 2011; 59 (6): 9809886.

8. De Benoist B et al. Worldwide prevalence of anemia 1993-2005. WHO Global Database on Anaemia Geneva. World Health Organization 2008.

9. Beghe C, Wilson A, Ershler WB. Prevalence and Outcomes of Anemia in Geriatrics: A Systematic Review of the Literature. Am J Med 2004;116(7A):310 .

10. Gaskell H, Derry S, Moore RA, McQuay HJ. Prevalence of anemia in older persons: systematic review. BMC Geriatrics 2008; 8:1.

11. Eisenstaedt R, Penninx BW, Woodman RC. Anemia in the elderly: Current understanding and emerging concepts, Blood Reviews 2006; 20(4): 213-226.

12. Price EA, Mehra R, Holmes TH, Schrier SL. Anemia in older persons: Etiology and evaluation, J BLOOD CELL MOL DIS 2011; 46:159-165.

13. Gabrilove J. Anemia and the elderly: clinical considerations, J Best Practice \& Research Clinical Haematology 2005; 18 (3): 417-422.

14. Bross MH, Soch K, Smith-Knuppel T. Anemia in Older Persons, Am Fam Physician 2010; 82(5):480-487.

15. Penninx BWJH, Guralnik JM, Onder G. Anemia and Decline in Physical Performance among Older Persons. Am J Med 2003; 115(2): 104-110.

16. Guralnik JM, Simonsick EM, Ferrucci L et al. A short physical performance battery assessing lower extremity function: Association with self-reported disability and prediction of mortality and nursing home admission. J Gerontol 1994; 49:85-94.

17. Lemmink KAP, Kemper H, de Greef MHG et al. Reliability of the Groningen fitness test for the elderly. J Aging Phys Activity 2001; 9:194-212.

18. Mahoney FI, Barthel DW. Functional evaluation: The Barthel Index. MD State Med J 1965; 14:61-65.

19. Yesavage JA, Brink TL, Rose TL et al. Development and validation of a geriatric depression screening scale: A preliminary report. J Psychiatr Res 1982; 17:37-49.

20. de Craen AJ, Heeren TJ, Gussekloo J. Accuracy of the 15-item geriatric depression scale (GDS-15) in a community sample of the oldest old. Int J Geriatr Psychiatry 2003; 18:63-66.

21. Tombaugh JA, Mcintyre NJ. The Mini-Mental State Examination: A comprehensive review. J Am Geriatr Soc 1992; 40:922-935.

22. Speer DC, Greenbaum PE. Five methods for Computing Significant Individual Client Change and Improvement Rates: Support for Individual Growth Cure Approach. J CONSULT CLIN PSYCH 1995; 63(6): 1044 - 1048.

23. Vellas B, Villars H, Abellan G et al. Overview of the MNA FIts history and challenges. J Nutr Health Aging 2006; 10:456-463.

24. Guigoz Y, Vellas B. The Mini Nutritional Assessment (MNA) for grading the nutritional state of elderly patients: Presentation of the MNA, history and validation. Nestle Nutr Workshop Ser Clin Perform Prog 1999; 1:3-11.

25. Ble A, Fink JC, Woodman RC, Klausner MA, Windham BG, Guralnik JM, Ferrucci L. Renal function, erythropoietin, and anemia of older persons: the InCHIANTI study. Arch Intern Med. 2005; 165(19):2222-7.

26. Thaler-Kall K, Döring A, Peters A, Thorand B, Grill E et al. Association between anemia and falls in community-dwelling older people: crosssectional results from the KORA-Age study. BMC Geriatrics 2014; 14:29.

27. Denny SD, Kuchibhatla MN, Cohen HJ. Impact of anemia on mortality, cognition, and function in community-dwelling elderly. Am J Med 2006; 119:327-334.

28. Riva E, Tettamanti M, Mosconi P, Apolone G et al. Association of mild anemia with hospitalization and mortality in the elderly: the Health and Anemia population-based study. J Haematol 2009; 94(1): 22-28.

29. Andrès E, Serraj K, Federici L, Vogel T, Kaltenbach G. Anemia in elderly patients: New insight into an old disorder. Geriatr Gerontol Int 2013; 13(3):519-527.

30. Gabrilove J. Anemia and the elderly: clinical considerations. J Best Practice \& Research Clinical Haematology 2005; 18 (3): 417-422.

31. Chaves PHM, Xue Q-L, Guralnik JM, Ferrucci L, Volpato S, Fried LP. What constitutes normal hemoglobin concentration in community-dwelling disabled older women? J Am Geriatr Soc 2004; 52:1811-1816.

32. Patel KV, Harris TB, Faulhaber M, Angleman SB, Connelly S, Bauer DC, et al. Racial variation in the relationship of anemia with mortality and mobility disability among older adults. J Blood 2007; 109:4663-4670.

33. Price EA, Mehra R, Holmes TH, Schrier SL. Anemia in older persons: Etiology and evaluation, Blood Cell Mol Dis 2011; 46:159-165.

34. Chaves PH, Ashar B, Guralnik JM, Fried LP. Looking at the relationship between hemoglobin concentration and prevalent mobility difficulty in older women. Should the criteria currently used to define anemia in older people be reevaluated? J Am Geriatr Soc 2002; 50(7):1527-1564.

35. Patel KV, Guralnik JM. Prognostic implications of anemia in older adults. Haematologica 2009; 94(1): 1-2.

36. Alemán H, Esparza J, Ramirez FA, Astiazaran H, Payette H. Longitudinal evidence on the association between interleukin- 6 and C-reactive protein with the loss of total appendicular skeletal muscle in free-living older men and women, Age Ageing 2011; 40(4):469-475.

37. Maccio A, Madeddu C. Review Article Management of Anemia of Inflammation in the Elderly 2012; 2012: 1-20.

38. Cesari M, Penninx BW, Pahor M, Lauretani F, Corsi AM, Rhys Williams G, Guralnik JM, Ferrucci L. Inflammatory markers and physical performance in older persons: the InCHIANTI study. J Gerontol A Biol Sci Med Sci 2004; 59(3):242-8

39. Visser M, Pahor M, Taaffe DR, et al. Relationship of interleukin-6 and tumor necrosis factor-alpha with muscle mass and muscle strength in elderly men and women: the Health ABC Study. J Gerontol A Biol Sci Med Sci 2002; 57: 326-332.

40. Cesari M, Penninx BW, Newman AB, Kritchevsky SB et al. Inflammatory markers and onset of cardiovascular events: results from the Health $A B C$ study, Circulation 2003; 108(19):2317-2322.

41. Brinkley TE, Leng X, Miller ME, Kitzman DW, Pahor M, Berry MJ, Marsh $\mathrm{AP}$ at al. Chronic Inflammation Is Associated With Low Physical Function in Older Adults Across Multiple Comorbidities, J Gerontol A Biol Sci Med Sci 2009; 64A(4): 455-461.

42. Culleton BF, Manns BJ, Zhang J, Tonelli M, Klarenbach S, Hemmelgarn BR. Impact of anemia on hospitalization and mortality in older adults. Blood 2006; 107(10):3841-3846.

43. Kaptoge S, Di Angelantonio E, Lowe G, Pepys MB, Thompson SG, Collins R, Danesh J. C-reactive protein concentration and risk of coronary heart disease, stroke, and mortality: an individual participant meta-analysis. Lancet 2010; 375(9709):132-140

44. Zakai NA, Katz R, Hirsch C, Shlipak MG et al. A Prospective Study of Anemia Status, Hemoglobin Concentration, and Mortality in an Elderly Cohort The Cardiovascular Health Study. Arch Intern Med 2005; 165(19):2214-2220

45. Harris TB, Ferrucci L, Tracy RP, Corti MC, Wacholder S, Ettinger WH Jr, Heimovitz H, Cohen HJ, Wallace R. Associations of elevated interleukin-6 and C-reactive protein levels with mortality in the elderly. Am J Med 1999; 106(5):506-512. 